Je vous prie de m'excuser d'avoir été souffrant ce matin. J'ai décidé que lorsque je serai à nouveau malade, je reviendrai à Paris.

Je ne vais pas traiter des raisons de la privatisation des services de l'eau, mais de l'environnement économique dans lequel va fonctionner l'industrie privatisée, et des conséquences de la privatisation.

En fait, cette privatisation ne concerne que l'Angleterre et le Pays de Galles. Les structures publiques existantes en Ecosse et en Irlande du Nord ne sont pas modifiées. Nous partons à l'origine de la situation britannique typique des "chambrers" avec la particularité suivante: il existe des compagnies privées de distribution d'eau, dont beaucoup ont été créées il y a une centaine d'années.

La privatisation n'a concerné que les anciennes Water Authorities (Autorités de l'Eau), à compétence régionale ou de bassin, qui ont été divisées en deux organismes :

- les Water Services (Services de l'Eau), sociétés à responsabilité limitée, qui ont compétence en matière d'assainissement et de distribution d'eau, lorsque cette distribution n'est pas assurée par l'une des compagnies privées pré-existantes; - les parties des Water Authorities qui avaient en charge la protection contre les crues et le contrôle de la pollution, regroupées au sein de la National Rivers Authority (Autorité Nationale des Cours d'Eau) et qui remplissent ces missions au titre du Ministère de l'Environnement.

De surcroît a été créé le Office of Water Services (Bureau des Services de l'Eau, sous la responsabilité du Director General of Water Services), chargé du contrôle des prix et des niveaux de qualité de service.

Pour les autres privatisations de services publics, le gouvernement a mis en place le contrôle des prix suivant: l'augmentation est

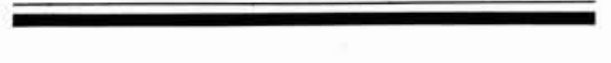

Expose de Monsicur Colin H. GREEN

(Traduction de M. J.P. TORTEROTOT)

Flood Hazard Research Centre, Middlesex Polytechnic, Enfield, Royaume-Uni

\section{FLASH SUR LA PRIVATISATION DES SERVICES DE L'EAU EN ANGLETERRE ET AU PAYS DE GALLES}

limitée au taux de l'inflation auquel on ote un certain pourcentage, ce pourcentage devant encourager aux gains de productivité. Le problème rencontré par le gouvernement dans la privatisation de l'industrie de l'eau, est que l'on ne pouvait pas procéder de même. L'industrie de l'eau est confrontée à une très faible croissance de la demande, mais aussi à de très importants besoins d'investissements pour l'amélioration de la qualité: probablement de l'ordre de 40 milliards de Livres Sterling pour les 20 prochaines années, à comparer à une valeur comptable actuelle des immobilisations de 15 à 18 milliards de Livres (estimée à la valeur de remplacement). Ceci a conduit à retenir, pour l'augmentation des prix, un taux maximum égal au taux d'inflation plus $\mathrm{K}$. Ce taux $\mathrm{K}$ est fixé de sorte à permettre l'accroissement des investissements, et sa valeur est comprise entre 4,5 et 7 pourcents par an. De plus, il existe une possibilité de dépasser ce taux d'augmentation: le taux $\mathrm{K}$ est déterminé sur la base des besoins en investissements prévus, mais des besoins imprévus pourront se traduire par une augmentation plus importante des prix (après justification auprès du Bureau des Services de l'Eau).

La complexité de cette formule d'encadrement des prix, ainsi que ses retombées inflationnistes, vont à mon avis conduire à ce que finalement le gouvernement intervienne plus qu'avant dans la fixation des prix, et de façon plus détaillée. Ceci malgré le fait que pour l'industrie de l'eau, l'avantage attendu de la privatisation était de se débarrasser de la tutelle gouvernementale.

Ainsi, je pense que les conséquences de la privatisation de l'industrie de l'eau sont négatives ou neutres en termes d'efficacité économique. Mais le mode d'amélioration de l'efficacité économique que recherche en fait le gouvernement, c'est l'internalisation par -l'industrie de l'eau- des coûts sociaux. Jusqu'ici, nous en avons observé les prémisses dans deux domaines. Premièrement, le gouvernement est en train de se donner la possibilité de faire payer aux services publics les coûts de l'excavation des routes. Deuxièmement, on a commencé à taxer les autorisations de rejets d'eaux usées dans les cours d'eau. Et selon moi il est probable qu'à long terme, ces taxes soient fixées sur la base des impacts que les rejets ont sur les cours d'eau.

Par conséquent, je pense qu'il est encore tôt, moins de six mois après la privatisation, pour dire comment tout cela va fonctionner. C'est une expérience intéressante. Avant la privatisation, on recherchait l'efficacité économique par l'intégration des fonctions de distribution d'eau et d'assainissement. Maintenant, je pense qu'on va rechercher l'efficacité économique au travers des mécanismes de tarification.

(cette traduction n'ayant pas pu être contrôlée par Monsieur C.H. GREEN, les erreurs éventuelles de transcription et de traduction seraient de la responsabilité du traducteur)

\title{
Discussion
}

\section{VIGNOLES}

M. Colin Green, nous vous remercions. Dans la salle, il y a sûrement des questions à poser, je vais vous passer la parole, après avoir fait remarquer qu'il sera probablement intéressant de suive la privatisation également de la Ville de Toulouse. Je ne sais pas si je ne vais pas proposer une mission à M. Colin Green, parce que les évolutions sont tout à fait parallèles. Y-a-t'il des questions, des informations supplémentaires que vous souhaiteriez avoir?

\section{Mme GOGEZ}

Peut-on savoir un peu mieux comment sont organisés les rapports entre des administration chargées des problèmes de l'eau et des organismes privés ?

\section{GREEN}

Je pense que cela va être compliqué. Avant la privatisation, les défenseurs de l'environnement, qui sont très puissants et le deviennent de plus en plus au RoyaumeUni, craignaient que la National Rivers Authority (NRA) ne manque vraiment de pouvoirs. Mais en fait, la NRA va etre puissante, et il faudra qu'elle le soit politiquement. Ainsi, on a d'un côté la NRA qui va füxer les taxes imposées sur les rejets. Et de l'autre coté, il y a l'industrie, à la fois l'industrie de l'eau et les industries qui rejettent des polluants. Le Bureau des Services de l'Eau va essayer de faire tenir l'édifice. C'est comme si vous cherchiez à mettre ensemble trois furets dans un sac. 


\section{BARRAQUE}

Je voudrais savoir comment les ingénieurs, les professionnels de l'eau vivent cette situation? Sont-ils satisfaits ? Quels sont leurs sentiments vis à vis de cette privatisation, très surprenante pour des observateurs par rapport à la culture de l'eau en Angleterre?

\section{GREEN}

Je pense que l'on peut dire avec impartialité que beaucoup des techniciens de l'eau n'appréciaient pas du tout lídée de privatisation, parce quils ont une culture de service public. Et on leur demande de se placer dans une situation où les services sont fournis dans un but de profit. Beaucoup d'entre eux y sont opposés.

Je vais expliquer comment a été constituée la National Rivers Authority. Cette NRA a existé d'abord à l'échelon régional, les Water Authorities ayant été divisées avant la privatisation: d'un coté les personnes qui iraient dans les services de l'eau privés, de l'autre celles qui constitueraient l'échelon régional de la NRA. Je pense que, dans une certaine mesure, les gens qui se sentaient le plus attachés aux missions de service public ont rejoint la NRA. Mais cela soulève le problème de la façon dont la NRA a été dotée en personnel. Dans une certaine mesure, les parties des Water Authorities qui allaient devenir les compagnies privées ont décidé quels personnels rejoindraient la NRA. Ainsi, elles ont pu dire jusqu'à un certain point: celui-ci n'est pas bon, envoyons-le à la NRA, mais gardons celui-la, car il est bon. Et on peut observer que, contrairement d̀ ce qui se passe le plus souvent, les personnes compétentes n'ont pas pu choisir et n'ont donc pas été autorisées à rejoindre la NRA.

\section{BARRAQUE}

La privatisation des Water Authorities va-t-elle dans le sens d'une centralisation encore plus poussée de la gestion de l'eau en Grande Bretagne, ou plutot dans le sens d'une décentralisation par rapprochement des collectivités locales?

\section{GREEN}

A la base, la théorie était idéologique: la privatisation est une bonne chose, et on va privatiser les Water Authorities. Le problème était alors d'élaborer une procédure permettant de réaliser la privatisation de fagen légale. Ainsi, on n'a pas fait l'absurdité d'invoquer nos principes fondamentaux de décentralisation ou de centralisation. En Grande-Bretagne, nous n'avons pas de politiques, nous n'avons que des gesticulations politiques. En pratique, aucun gouverne- ment ne pourra laisser passer les effets inflationnistes de la formule d'encadrement des prix, qui autorise une augmentation supérieure de 4,5 à 7 pourcents au taux de l'inflation. Et il y aura une forte pression gouvernementale pour maintenir les prix à un niveau modéré.

Deuxièmement, dans le cadre du mouvement vers la privatisation, le gouvernement a supprimé il y a cinq ans la représentation des élus locaux au sein des Water Authorities (représentation instituée lors de leur création). Les Water Authorities furent alors exclusivement dirigées par des hommes d'affaires. Les conseils d'administration ne furent plus publics, mais tenus à huisclos. Ainsi, on s'est éloigné de toute participation locale aux responsabilités.

Un autre point important mérite, d̀ monvavis, d'être mentionné: jusquá la privatisation, le gouvernement ne fixait pas seulement les prix, mais également les limites d'investissements et d'endettements des Water Authorities. Pour cette raison, le gouvernement se devait de couvir les Water Authorities: si quelqu'un se plaignait de la qualité des cours d'eau, le Ministre devait répondre devant le Parlement et dire que tout se passe magnifiquement bien. Dans le cas contraire, il aurait fallu avouer que l'on n'investissait pas suffisamment. $A$ présent, avec la privatisation, l'industrie de l'eau a perdu la protection apportée par le gouvernement. Si quelqu'un se plaint pour un motif donné, le Ministre va se lever et dire: "nous n'avons pas privatisé l'industrie de l'eau pour que ceci arrive, quelque chose doit être fait.

\section{D'ASSONVILLE}

Existe-t-il des dispositits concernant les consommateurs?

\section{GREEN}

II existe principalement des comités consultatifs de consommateurs. II existait déjà de tels comités auprès des Water Authorities, pour être informés plutot que pour décider. Et je soupfonne qu'ils vont être encore moins influents à présent, dans la nouvelle structure.

\section{BACHOC}

Peut-on déjà constater que la privatisation a tendance à influencer la répartition des compétences à l'intérieur des établissements privés qui s'occupent de l'eau?

Voit-on, par exemple, se modifier la proportion des gens qui s'occupent d'encadrement et d'exécution, ou voit-on plus facilement s'opérer une technicisation des services ? Etant donné qu'en France, l'une des motivations pour la privatisation est que le statut de la fonction territoriale est plutot un handicap pour adapter les services aux nouvelles tâches qui les attendent.

\section{GREEN}

Dans le cadre du mouvement vers la privatisation, les Water Authorities avaient réduits leur personnel de façon assez drastique. Et à présent, dans la mesure où il est possible de faire des pronostics, les services vont sous-traiter une plus grande part de leurs activités, en particulier de leurs activités de conception. Ceci malgré le fait qu'ils cherchent à développer leur capacité de consultants. J'entrevois deux tendances. Premièrement, il y a à mon avis des signes montrant que le potentiel humain a déjà été trop réduit pour pouvoir utiliser efficacement les équipements. Et deuxièmement, il y a des signes indiquant que les services de l'eau vont tenter de réaliser moins d'activités dans leurs propres murs, et recourir plus à des consultants et prestataires extérieurs.

\section{OLIVA}

Vous nous avez donné des indications générales sur l'évolutions des coûts de l'assainissement avec la privatisation, jaurais aimé avoir des précisions selon les différents types d'usagers, c'est-à-dire, si l'on se place dans le cas d'une collectivité locale, dans le cas d'une personne privée, ou dans le cas d'une entreprise.

Une autre question qui vient à l'esprit est quavec la privatisation, on considère des secteurs qui sont rentables et d'autres qui ne le sont pas, par exemple, les grands travaux et l'entretien; si l'on prend, par exemple, l'entretien, quelles sont les conséquences en terme d'emploi et, à terme, en matière de qualité du service rendu ?

Enfin, dernière question, quelle influence budgétaire cela a-t-il sur l'état, parce que naivement, on pourrait se dire, voilà quelque chose qui est rendu au privé, cela va nous faire diminuer nos impots, ou au contraire, les impots globalement restent les mémes et finalement, on arrive d̀ un doublement des charges pour tout le monde?

\section{GREEN}

Tout d'abord, la réponse à la question sur la tarification. Pour la consommation domestique, dans la situation actuelle, les tarifications de la distribution d'eau comme de l'assainissement sont basées sur la taxe immobilière. Plus la valeur du logement est élevée, plus vous devrez payer. Ceci va changer, et des expériences de comptage de l'eau sont en cours. Ceci va changer, parce que le gouvernement a annoncé que cela 
allait changer. Mais le comptage va poser des problèmes. Actuellement, il semble que sur le plan économique, le coût du comptage excède les économies qu'il induit, et il existe également des problèmes d'ordre technique. Mais on ne sait pas ce qu'il adviendra dans le futur.

En ce qui concerne les consommateurs industriels, la taxation des rejets dans lesystème d'assainissement est déterminée par la "formule de MOGDEN", formule agréée par la Confédération Britannique de I'Industrie et les Water Authorities. Cette formule, qui fixe les taxes par rapport au volume et au contenu de l'effluent, est supposée se baser sur les coûts de traitements.

Venons à la seconde question. L'une des fonctions du Bureau des Services de l'Eau, à coté de la füxation des prix, est la fïxation des niveaux de qualité de service. II s'agit de s'assurer que les compagnies d'eau ne fassent pas de profits en réduisant la maintenance. $\mathrm{La}$ fixation de niveaux de service est une question difficile, lorsqu'il s'agit de la traduire par des normes de maintenance. Cela étant, beaucoup de gens affirment que le niveau actuel de la maintenance est si bas que cela ne pourrait pas devenir pire!

L'une des majeures raisons de la privatisation, est que les emprunts des Water Authorities étaient comptabilisés comme des emprunts de l'Etat, ceciafin de pouvoir controler les emprunts du secteur public. La privatisation a rapporté 1,5 milliards de Livres. Mais pour pouvoir encaisser cela, le gouvernement a dû effacer 4 milliards de Livres de dettes en souffrance, et injecter 1,5 milliards d'argent frais, pour rattraper le retard d'investissements. II faut ajouter à cela environ une centaine de millions de Livres dépensées pour mettre en place la privatisation. A long terme, il y aura unc réduction des impots, du fait de la taxation des compagnies. Mais il est difficile d'estimer cette réduction.

\section{VIGNOLES}

II nous faut maintenant passer $\dot{a}$ un autre sujet, je vais donc remercier $M$. Colin Green et remercier M. Torterotot, qui a réussi un exercice de traduction toujours périlleux. Merci pour la qualité de leur exposé.

Je vais demander à M. Brigando de venir prendre leur suite sur les aspects économiques de lassainissement pluvial.

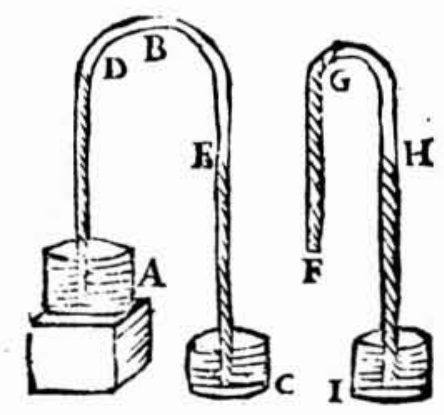

